\title{
Nanofibers: Efficient Material for Dye Removal Applications
}

\author{
Zeeshan Khatri, Umair Ahmed, Qureshi and Farooq Ahmed
}

\begin{abstract}
Development of green method for controlling dye pollution in water is currently a challenging issue. Most of the methods reported are either expensive or inefficient to meet the standard quality of usable water. Therefore, we attempt to prepare highly efficient and environmental friendly nanofiber membranes for the removal of anionic dyes as well as cationic dyes through batch adsorption method. These nanofibers proved to be promising adsorbents as they required minimum adsorption time, normal working $\mathrm{pH}$, ambient temperature and minimum adsorbent dosage. The adsorption data fitted well with pseudo-second order. The proposed nanofibers are easy to prepare and have no bad impact on environment.
\end{abstract}

Keywords-Nanofibers; adsorption; dyes; electrospinning;water treatment.

\section{INTRODUCTION}

Dye contamination is a critical global issue. Textile industry consumes significant amount of water during dyeing and finishing operations which may generate greater effluents containing these dyes. The presence of these dyes in water not only prevents photosynthetic activities in plants through inhibition of sunlight penetration but also tends to cause many poor health impacts such as skin irritation, respiratory problems and carcinoma [1]. Due to their complex chemical structures, these dyes are recalcitrant to degrade through light or microorganisms. Although a variety of techniques such as microbial degradation, membrane filtration, advanced oxidation processes and electrochemical process is available to treat these toxic dyes but such methods are limited with cost, energy and efficiency [2]. Some methods require using oxidants that are themselves hazardous to human health, some methods generate sludge or cause membrane fouling. Besides these methods, adsorption is considered simple, easy and low cost technique. It offers many advantages such as variety of materials, interesting features of desired adsorbent can be achieved by tuning surface properties of materials, easy surface modification to transform hydrophilic to hydrophobic characteristics and vice versa [3].

Zeeshan Khatri, Umair Ahmed Qureshi and Farooq Ahmed Nanomaterials Research Lab, Department of Textile Engineering, Mehran University of Engineering and Technology, Jamshoro, 76060, Pakistan.
Different materials either natural or synthetic have been reported for dye adsorption depending upon properties of dyes being tested. Some of the materials include carbon nanotubes, grapheme oxide, magnetic activated carbons, synthetic resins, modified resins, chitosan and cellulose based adsorbents [4-6]. Such materials have been tested in powder form that requires filtration step for separation after adsorption experiment. This process may also lead loss of some spent sample for regeneration purpose and also tend to cause secondary pollution through sludge generation. Moreover, the above mentioned materials require either prolonged synthesis steps or proved low efficiency removal to target dye. Nowadays, nanotechnology particularly one dimensional nanosized membrane known as nanofibers has grasped this problem by preventing filtration step and enhanced adsorption efficiency due to high surface to volume ratio.

In present work, our group has prepared some highly efficient nanofiber adsorbents from cheap and abundantly available polymers for the removal of some anionic and cationic dyes. Our newly prepared nanofiber membranes not only save adsorption processes time but also donot require any pre or post chemical modification unlike other materials reported previously.

\section{II.PROCEDURE}

\section{Preparation of Nanofibers}

\section{A. Preparation of nylon-6 nanofibers}

A $22 \%$ (w/v) nylon-6 solution was prepared by dissolving known quantity of nylon-6 in formic acid solvent for $2 \mathrm{~h}$. The resulting solution was casted in plastic syringe (i.d $0.6 \mathrm{~mm}$ ). The syringe was placed opposite to ground collector. Electrospinning was performed by applying potential of $20 \mathrm{kV}$ between positively charged terminal (connected to plastic syringe) and negative charged terminal (connected to ground collector). After completion of electrospinning process, the sample was dried overnight at room temperature.

\section{B. Preparation of Zein/Nylon coelectrospun nanofibers}

Nylon-6 solution was prepared and electrospun with zein by the method discussed above. 60\% Zein (w/v) solution was prepared in DMF. This solution along with nylon-6 solution was electrospun through the similar procedure used for nylon6 


\section{Preparation of carbon nanofibers}

For the preparation of carbon nanofibers, its precursor i.e. $10 \%(\mathrm{w} / \mathrm{v})$ polyacrylonitrile (PAN) solution in DMF was electrospun. The electrospinning parameters were relatively similar to the above mentioned methods. After PAN electrospun nanofibers were achieved the sample left to dry overnight at room temperature. The electrospun nanofibers were then heated at $280^{\circ} \mathrm{C}$ with a heating rate of $3^{\circ} \mathrm{C} / \mathrm{min}$ and 1 hour hold time at $280 \mathrm{oC}$ to carry out stabilization process. Carbonization and activation of heated nanofibers was carried out by our own developed method called plate sandwich method in which nanofiber sheet was placed in between two rectangular stainless steel plates tighten by nuts and bolts on all four sides. Plates were filled with the sand to avoid oxidation conditions. The plates were then heated in muffle furnace at $600^{\circ} \mathrm{C}$ with a heating rate of $5^{\circ} \mathrm{C}$ and 1 hour hold time at specified temperature. Carbon nanofibers (CNF) were finally obtained and characterized.

\section{ADSORPTION EXPERIMENT}

\section{Batch Adsorption Technique}

Three different types of dyes, i.e. two anionic dyes (Acid Blue 117 and Reactive Blue 19) and one cationic dye (Methylene Blue) were chosen for adsorption study. Different parameters such as $\mathrm{pH}$ (1-11) and time (1-60 $\mathrm{min}$ ) for adsorption were optimized by taking fixed mass of nanofibers (20mg) at room temperature. The samples were shaken at 200 rpm until equilibrium was achieved. The adsorption efficiency of the nanofibers was calculated through the absorbance values obtained before and after adsorption of different dyes by means of UV-Vis spectrophotometer according to the relation

$$
\text { Adsorption Efficiency } \%=\frac{\left(C_{i}-C_{f}\right)}{C_{i}} \times 100
$$

Where $\mathrm{Ci}$ refers to the initial dye concentration $(\mathrm{mg} / \mathrm{L}), \mathrm{Cf}$ refers to the final dye concentration $(\mathrm{mg} / \mathrm{L})$ after adsorption, $\mathrm{V}$ is the volume of the dye solution (L), $\mathrm{m}$ is the mass of nanofiber $(\mathrm{g})$.

\section{RESUlts AND Discussion}

\section{Characterization}

The SEM images of neat Zein, Nylon-6, composite of zein/nylon, PAN and carbon nanofibers is presented in Fig-1 (a, b, c,d and e). The morphology of all nanofibers was bead free and smooth. The average mean diameter of zein, nylon-6 and zein/nylon nanofiber were found to be 130, 100, $150 \mathrm{~nm}$ respectively, while that of PAN and carbon nanofibers was 315 and $250 \mathrm{~nm}$ respectively., clearly state the units for each quantity in an equation.
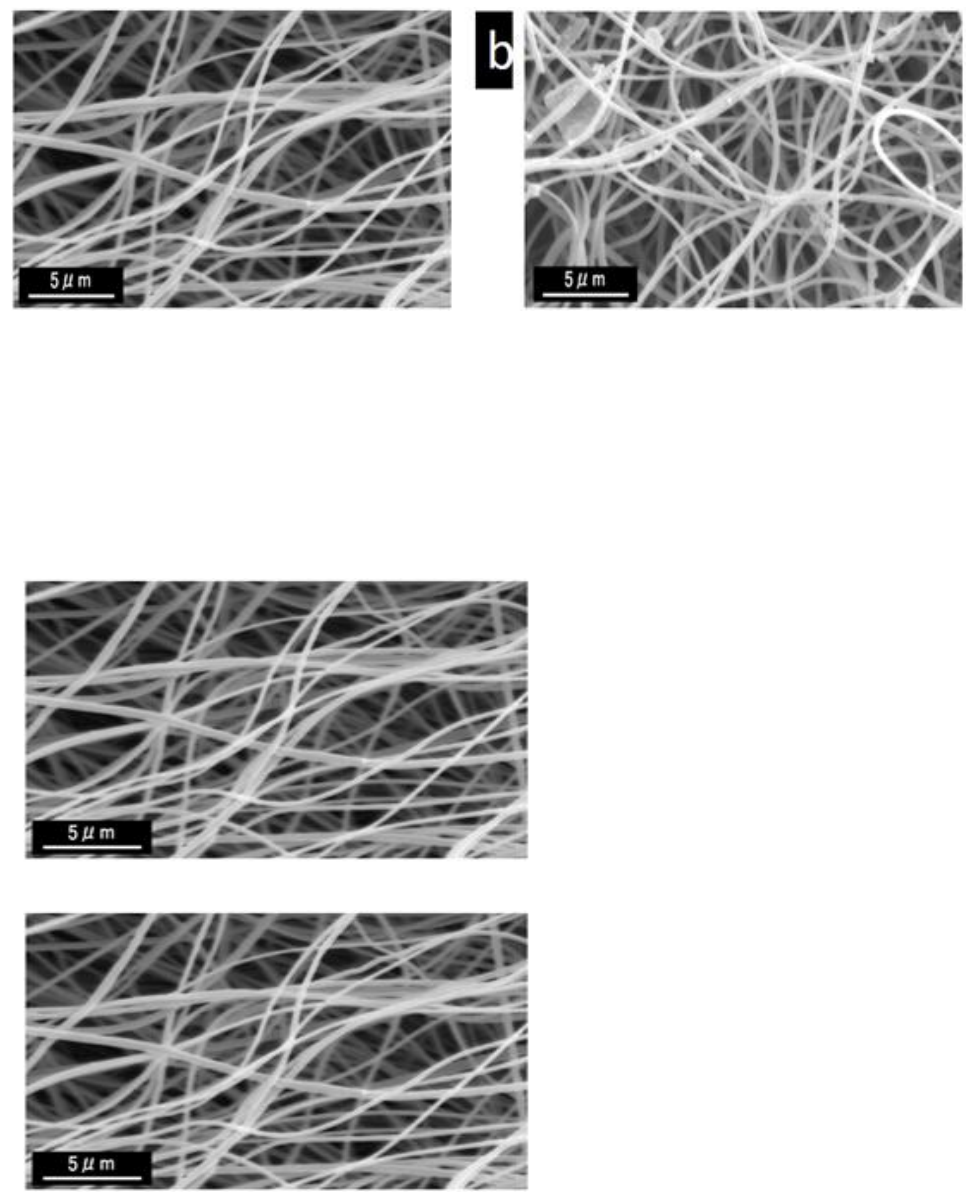

Fig. 1 SEM images of (a) Zein nanofibers, (b) Nylon-6 nanofibers, (c) Zein/Nylon-6 nanofibers, (d) PAN nanofibers and (e) carbon nanofibers.

\section{Adsorption Study}

Adsorption study was undertaken to examine the optimum time for maximum adsorption of Acid blue 117, reactive blue 19 and methylene blue dye. The appropriately measured mass of nanofibers was taken in small test tube containing $50 \mathrm{mg} / \mathrm{L}$ each dye in a separate tube. The $\mathrm{pH}$ of adsorption medium was around 5. The samples were shaken at given intervals and the absorbances values were measured with the help of UV-Vis spectrophotometer. It was found that adsorption efficiency increase as the time of contact between nanofibers and dyes was increased. This may be due to proper contact between two interfaces with the passage of time. More dye molecules can interact. After some time, further adsorption cannot be noticed probably due to saturation of vacant sites that may lead to plateau in the curve. The optimum time for acid blue 117 was found $25 \mathrm{~min}$, that of reactive blue 19 was $5 \mathrm{~min}$ and that of methylene blue was 15 min using nylon-6, zein nylon-6 and carbon nanofibers respectively. 

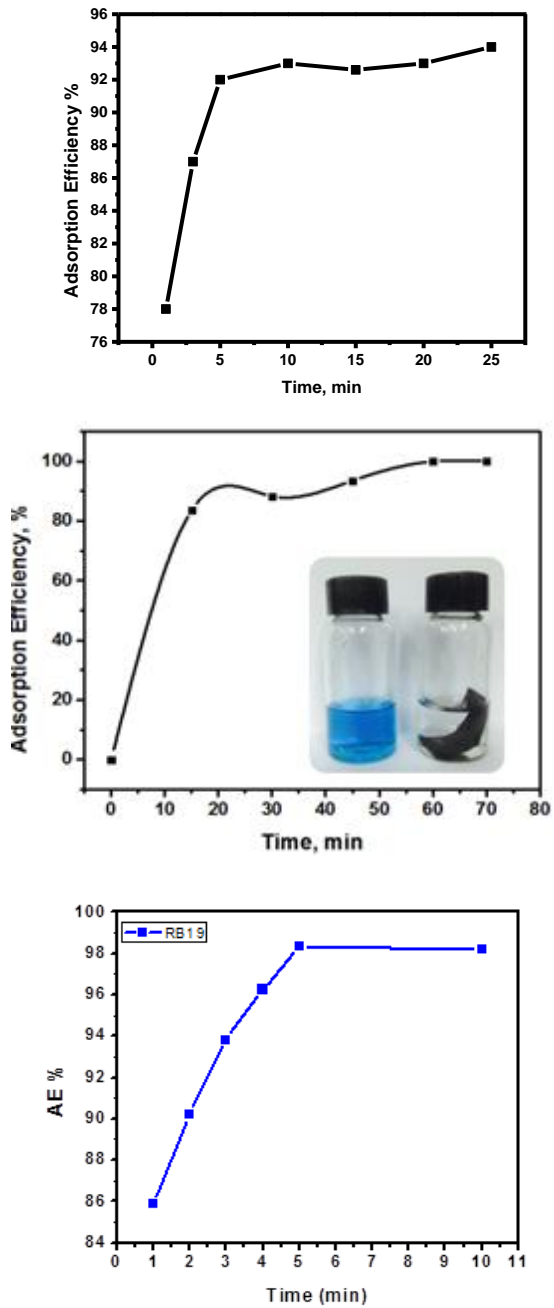

Fig.2 Effect Of Time (Min) On Adsorption Efficiencies Of (A) Nylon-6 For Acid Blue 117 Removal, (B) Zein/Nylon-6 On Reactive Blue Dye Removal And (C) Carbon Nanofibers On Methylene Blue Dye Removal.

From the above results, it is evident that the current nanofibers offer minimum possible time for complete dye removal as compared to previously used materials. Theses nanofibers are not only easy to fabricate and use but also offer less time in removal of different dyes. This behavior may be attributed to enhanced surface area and highly porous structure of nanofiber membrane. Table 1. compares the adsorption capacities and time taken by different materials for the studied dyes. The proposed nanofibers show worth time saving and higher capacities
TABLE 1. COMPARISON OF ADSORPTION CAPACITIES AND OPERATION TIME OF CURRENT NANOFIBERS WITH PREVIOUSLY REPORTED MATERIALS

\begin{tabular}{|c|c|c|c|}
\hline \multicolumn{4}{|l|}{ Acid Blue } \\
\hline Adsorbent & $\begin{array}{l}\text { Adsorption } \\
\text { capacity } \\
(\mathrm{mg} / \mathrm{g})\end{array}$ & $\begin{array}{l}\text { Time } \\
(\mathrm{min})\end{array}$ & Reference \\
\hline Nylon-6 & 58.8 & 25 & Present study \\
\hline $\begin{array}{l}\text { Activated carbon } \\
\text { from rubber tyre }\end{array}$ & 9.2 & 40 & [7] \\
\hline Chitin gels & 40 & 1200 & {$[8]$} \\
\hline \multicolumn{4}{|l|}{ Reactive Blue 19} \\
\hline Adsorbent & $\begin{array}{l}\text { Adsorption } \\
\text { capacity } \\
\text { (mg/g) }\end{array}$ & $\begin{array}{l}\text { Time } \\
\text { (min) }\end{array}$ & Reference \\
\hline Zein/Nylon-6 & 70 & 5 & Present study \\
\hline $\mathrm{NiO}$ nanoparticles & 98.8 & 120 & [9] \\
\hline $\begin{array}{l}\text { Magnetic } \\
\text { grapheme oxide }\end{array}$ & 62.5 & 66 & {$[10]$} \\
\hline \multicolumn{4}{|l|}{ Methylene Blue } \\
\hline Adsorbent & $\begin{array}{l}\text { Adsorption } \\
\text { capacity } \\
\text { (mg/g) }\end{array}$ & $\begin{array}{l}\text { Time } \\
\text { (min) }\end{array}$ & Reference \\
\hline $\begin{array}{l}\text { Carbon } \\
\text { nanofibers }\end{array}$ & 72.46 & 60 & \\
\hline $\begin{array}{l}\text { Carbonized citrus } \\
\text { fruit peel }\end{array}$ & 25.51 & $8 \mathrm{hr}$. & [11] \\
\hline $\begin{array}{l}\text { Fe3O4@ZIF-8 } \\
\text { core-shell } \\
\text { heterostructure }\end{array}$ & 20.20 & $15 \mathrm{hr}$. & {$[12]$} \\
\hline
\end{tabular}

\section{V.CONCLUSION}

The proposed nanofibers show high efficiency towards different cationic and anionic dyes. The maximum removal time was also minimum compared to other previously reported materials. The nanofiber based adsorbents offer many advantages such as easy separation, good removal efficiency within short time and no lengthy procedures for preparation.

\section{ACKNOWLEDGMENT}

The work was supported by Mehran University of Engineering and Technology Jamshoro

\section{REFERENCES}

[1] L.D Fiorentin,.; D.E.G Trigueros,.; A.N Modenes,; F.R. EspinozaQui nones,; N.C Pereira,.; Barros,; O.A.A Santos,. Biosorption of reactive blue $5 \mathrm{G}$ dye onto drying orange bagasse in batch system: Kinetic and equilibrium modeling. Chem. Eng. J. 2010, 163, 68-77 https://doi.org/10.1016/j.cej.2010.07.043

[2] S.Chakraborty,; S.Chowdhury,; P.Das Saha, Adsorption of crystal violet from aqueous solution onto $\mathrm{NaOH}$-modified rice husk, Carbohydr. Polym. 2011, 86, 1533-1541 https://doi.org/10.1016/j.carbpol.2011.06.058

[3] S.Zhu.; S.Jiao,; Z.Liu,; G.Pang and S.Feng, Hydrothermal reactions from carbon dioxide to phenol. Environ. Sci.: Nano, 2014, 1, 172.

[4] S.Li ,; Z.Jia,; Z.Li,; Y.Li,; R.Zhu,; Synthesis and characterization of mesoporous carbon nanofibers and its adsorption for dye in wastewater. Adv. Powder Technol. 2016, 27, 591-598 https://doi.org/10.1039/C3EN00078H.

[5] J.G.Lee, ; D.Y.Kim, ; M. G.Mali,; S. S. Al-Deyab, ; M. T. Swihart, and S. S. Yoon, . Supersonically blown nylon-6 nanofibers entangled with graphene flakes for water purification Nanoscale, 2015,7, 1902719035

https://doi.org/10.1016/j.apt.2016.01.024 
[6] K.Z. Elwakeel, Removal of Reactive Black 5 from aqueous solutions using magnetic chitosan resins J. HAZARD. MATER.167 (2009) 383392. https://doi.org/10.1039/C5NR06549F

[7] V.K.GUPTA,; B.GUPTA,; A.RASTOGI,; S.AGARWAL,; A.NAYAK, A comparative investigation on adsorption performances of mesoporous activated carbon prepared from waste rubber tire and activated carbon for a hazardous azo dye-Acid Blue 113 J. HAZARD. MATER. 2011, $186,891-901$.

https://doi.org/10.1016/j.jhazmat.2009.01.051

[8] L.VACHOUD,; N.ZYDOWICZ; A .DOMARD, Sorption and desorption studies on chitin gels INT. J. BIOL. MACROMOL. 2001, 28, 93-101 https://doi.org/10.1016/j.jhazmat.2010.11.091.

[9] MZ Khoshhesab, M Ahmadi removal of reactive blue 19 from aqueous solution using $\mathrm{NiO}$ nanoparticle Desalination and Water Treatment (2015) 57(42): 20037-20048,

https://doi.org/10.1016/S0141-8130(00)00151-3

[10] Z Ayazi, Khoshhes, S Norouzi MODELING AND OPTIMIZING OF ADSORPTION REMOVAL OF REACTIVE BLUE 19 ON THE MAGNETITE/GRAPHENE OXIDE NANOCOMPOSITE VIA RESPONSE SURFACE METHODOLOGY.Desalination and Water Treatment (2016) 57(52): 25301-25316. https://doi.org/10.1080/19443994.2015.1101713

[11] S.DUTTA, A.BHATTACHARYYA, A.GANGULY, S.GUPTA, \& S BASU, Application of Response Surface Methodology for preparation of low-cost adsorbent from citrus fruit peel and for removal of Methylene Blue.DESALINATION, (2011). 275(1), 26-36 https://doi.org/10.1080/19443994.2016.1157705

[12] J.Zheng, , C.Cheng, , W.-J.Fang, , C.Chen, , R.-W Yan,., H.-X.Huai, , \& C.-C.Wang, (2014). Surfactant-free synthesis of a Fe3O4@ZIF-8 coreshell heterostructure for adsorption of methylene blue.CrystEngComm, 16(19), 3960-3964 https://doi.org/10.1039/c3ce42648c

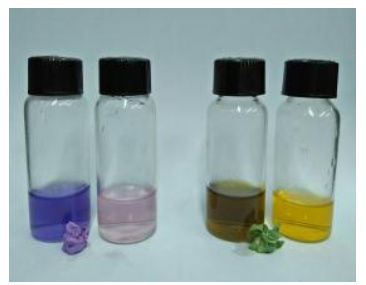

